\title{
The Time Evolution of Quantized Fields with Bounded Quasi-local Interaction Density
}

\author{
R. F. STREATER and I. F. WILDE* \\ Mathematics Department, Bedford College, London
}

Received September 11, 1969

\begin{abstract}
We extend to arbitrary dimension the proof by Guenin that the timeevolution is an automorphism group of the local algebras, if the interaction Hamiltonian is a space-integral of a bounded local density with finite range.
\end{abstract}

It has been suggested $[1,2]$ that, in order to avoid the divergences of quantum field theory, the time-evolution might be regarded as an automorphism group of some $C^{*}$-algebra $\mathfrak{A}$; if there is a non-trivial interaction, these automorphisms will not be implemented by unitary transformations in the "free" representation of $\mathfrak{A}$.

These ideas have been illustrated in a linear model [1], and in twodimensional relativistic theories with bounded interaction densities [3]. A similar result has been demonstrated for the Heisenberg ferromagnet and certain fermion systems $[4,5,8,9]$. In the present paper we offer a generalisation of some of the results of [3] and [5].

We work in the algebraic approach to quantum field theory [2]. More precisely, we make the following assumptions:

1. We are given a $B^{*}$-algebra $\mathfrak{A}$ of observables, and to each bounded open subset $\mathcal{O}$ of $\mathbb{R}^{4}$, we are given a sub- $B^{*}$-algebra $\mathfrak{A}(\mathcal{O})$; we assume that the various $\mathfrak{A}(\mathcal{O})$ generate $\mathfrak{A}$.

2. Causality: if $\mathcal{O}_{1}$ and $\mathcal{O}_{2}$ are space-like separated, then $\mathfrak{A}\left(\mathcal{O}_{1}\right)$ commutes with $\mathfrak{2}\left(\mathrm{O}_{2}\right)$.

3. Free field dynamics: we are given a continuous homomorphism, $\tau_{0}$, from $\mathbb{R}^{4}$ into the automorphism group of $\mathfrak{A}$, such that $\tau_{0}(a) \mathfrak{A}(\mathcal{O})$ $=\mathfrak{A}\left(\mathcal{O}_{a}\right)$, where $\mathcal{O}_{a}=\left\{x \in \mathbb{R}^{4} ; x-a \in \mathcal{O}\right\}$. By continuity we mean that $\left\|\tau_{0}(a) A-A\right\| \rightarrow 0$, for any $A \in \mathfrak{H}$, as $a \rightarrow 0$ in $\mathbb{R}^{4}$. For example, $\mathfrak{A}(\mathcal{O})$ could be the $C^{*}$-algebra generated by a free scalar field $\phi$, smeared with test-functions in $\mathscr{D}(\mathcal{O})$, or that generated by even powers of a free Dirac field in $\mathcal{O}$.

* Supported by the Science Research Council. 
Let $V \in \mathfrak{A}\left(\mathcal{O}_{1}\right)$ be any hermitian local observable, and let $V_{\boldsymbol{a}}=\tau_{0}(0, \boldsymbol{a}) V$. Then $\int_{|\boldsymbol{a}| \leqq r} V_{\boldsymbol{a}} d^{3} a=V_{r}$ may be defined (see [6]) by a Riemann-Bochner integral, and so defined, lies in $\mathfrak{A}$.

We also have that $\tau_{0}^{-1}(t, \mathbf{0}) V_{r} \equiv V_{r}(t)=\int_{|\boldsymbol{a}| \leqq r} V_{\boldsymbol{a}}(t) d^{3} a$.

We shall regard $V_{r}$ as an interaction potential; it perturbs the free dynamics $\tau_{0}(t)$ and defines a new time-evolution $\tau_{r}(t)$ (Theorem 1). The limit $r \rightarrow \infty$ exists (Theorem 2), defining a theory with space-translation symmetry (Theorem 3 ).

Let $A \in \mathfrak{A}$, and consider the following infinite sum,

$$
\tau_{r}^{I}(t) A=A+i \int_{0}^{t} d t_{1}\left[V_{r}\left(t_{1}\right), A\right]+i^{2} \int_{0}^{t} d t_{1} \int_{0}^{t_{1}} d t_{2}\left[V_{r}\left(t_{1}\right),\left[V_{r}\left(t_{2}\right), A\right]\right]+\cdots
$$

Now, $\tau_{0}^{-1}(t, \mathbf{0})$ is an automorphism and so $\left\|V_{r}(t)\right\|=\left\|V_{r}\right\|$, for every $t$, hence

$$
\left\|V_{r}(t)\right\|=\left\|V_{r}\right\| \leqq \int_{|\boldsymbol{a}| \leqq} d^{3} a\left\|V_{\boldsymbol{a}}\right\|=\frac{4}{3} \pi r^{3}\|V\|,
$$

since $\left\|V_{\boldsymbol{a}}\right\|=\|V\|, \tau_{0}(0, \boldsymbol{a})$ being an automorphism.

The $(n+1)^{\text {th }}$ term of the sum is a multiple commutator which can be expanded to give $2^{n}$ various permutations of $V_{r}\left(t_{1}\right) \ldots V_{r}\left(t_{n}\right) A$. The norm is therefore less than or equal to

$$
2^{n}\left\|V_{r}\right\|^{n}\|A\| \int_{0}^{|t|} d t_{1} \ldots \int_{0}^{t_{n}-1} d t_{n}=2^{n}\left\|V_{r}\right\|^{n}\|A\| \frac{|t|^{n}}{n !} .
$$

It follows that the sum converges in $\mathfrak{A}$ in norm, for all $t .\left(V_{r}, A \in \mathfrak{A}\right.$ and $\mathfrak{A}$ is norm complete.) We can, therefore, define $\tau_{r}^{I}(t)$ on $\mathfrak{A}$ by $(*)$. This describes the approximate dynamics in the interaction picture.

We shall now give some properties of $\tau_{r}^{I}(t)$.

(i) $\tau_{r}^{I}(t)(\lambda A+B)=\lambda\left(\tau_{r}^{I}(t) A\right)+\tau_{r}^{I}(t) B$, for all $A, B \in \mathfrak{A}$, and all $\lambda \in \mathbb{C}$.

(ii) $\left(\tau_{r}^{I}(t) A^{*}\right)=\left(\tau_{r}^{I}(t) A\right)^{*}$, for all $A \in \mathfrak{U}$. These are obvious.

(iii) If $A$ and $B$ are in $\mathfrak{A}$, then $\left(\tau_{r}^{I}(t) A B\right)=\left(\tau_{r}^{I}(t) A\right)\left(\tau_{r}^{I}(t) B\right)$.

Proof. By definition, $\tau_{r}^{I}(t) A=A+i \int_{0}^{t} d t_{1}\left[V_{r}\left(t_{1}\right), A\right]+\cdots$ which converges uniformly in $t$, for $t \in[-T, T]$, say. Let $s_{n+1}$ denote the sum of the first $n+1$ terms. Then we can differentiate $s_{n+1}$ term by term, with the result

$$
\left.\frac{d s_{n+1}}{d t}\right|_{t=\theta}=i\left[V_{r}(\theta), s_{n}(\theta)\right] .
$$

The r.h.s. converges uniformly in $\theta$, for $\theta \in[-T, T]$, as $n \rightarrow \infty$, which implies the existence of the limit of the 1.h.s. (all limits being norm limits 
in $\mathfrak{U})$. The two facts: $s_{n}$ converges uniformly in $[-T, T]$; and $s_{n}^{\prime}$ converges uniformly in $[-T, T]$, are sufficient to imply that the $\lim s_{n}$ is differentiable, with derivative equal to the limit of $s_{n}^{\prime}$, as $n \rightarrow \infty$, for $t \in[-T, T]$. Hence

$$
\left.\frac{d}{d t}\left(\tau_{r}^{I}(t) A\right)\right|_{t=\theta}=i\left[V_{r}(\theta), \tau_{r}^{I}(\theta) A\right]
$$

Similarly,

$$
\left.\frac{d}{d t}\left(\tau_{r}^{I}(t) B\right)\right|_{t=\theta}=i\left[V_{r}(\theta), \tau_{r}^{I}(\theta) B\right]
$$

Now (1) satisfies the initial condition $\left.\tau_{r}^{I}(t) A\right|_{t=0}=A$, a similar one holding for (2).

Moreover, $\left(\tau_{r}^{I}(t) A B\right)$ satisfies the differential equation

$$
\left.\frac{d}{d t}\left(\tau_{r}^{I}(t) A B\right)\right|_{t=\theta}=i\left[V_{r}(\theta), \tau_{r}^{I}(\theta) A B\right]
$$

with the initial condition

$$
\left.\tau_{r}^{I}(t) A B\right|_{t=0}=A B .
$$

Now

$$
\begin{aligned}
\frac{d}{d t}\left\{\left(\tau_{r}^{I}(t) A\right)\left(\tau_{r}^{I}(t) B\right)\right\}= & i\left[V_{r}(t), \tau_{r}^{I}(t) A\right] \tau_{r}^{I}(t) B \\
& +i \tau_{r}^{I}(t) A\left[V_{r}(t), \tau_{r}^{I}(t) B\right],
\end{aligned}
$$

where we have used Eqs. (1) and (2),

$$
=i\left[V_{r}(t),\left(\tau_{r}^{I}(t) A\right)\left(\tau_{r}^{I}(t) B\right)\right]
$$

with the initial condition

$$
\left.\left(\tau_{r}^{I}(t) A\right)\left(\tau_{r}^{I}(t) B\right)\right|_{t=0}=A B .
$$

However, given the differential equation

$$
\frac{d f(t)}{d t}=i\left[V_{r}(t), f(t)\right]
$$

together with a given initial condition, we can obtain the unique solution by iteration. Comparing Eqs. (5) and (6) with (3) and (4) (or by direct iteration of (5) with (6)) we obtain the required result.

The above remark concerning the uniqueness of the solution of the D.E. with given initial condition, further implies that $\tau_{r}^{I}(t)$ is one-one.

We can summarise the above by simply saying that $\tau_{r}^{I}(t)$ is a one-one, continuous, algebraic *-homomorphism of $\mathfrak{U}$ into itself. 
Theorem 1. If $\tau_{0}(t)$ is the given automorphism of $\mathfrak{A}$ (Postulate 3$)$, with $a=(t, 0,0,0)$, then for $A \in \mathfrak{A}$,

$$
\tau_{0}(s) \tau_{r}^{I}(s) \tau_{0}(t) \tau_{r}^{I}(t) A=\tau_{0}(s+t) \tau_{r}^{I}(s+t) A,
$$

i.e. $\tau_{0}(s) \tau_{r}^{I}(s)$ form a 1-parameter group of homomorphisms of $\mathfrak{A}$ into itself.

Proof. Let $A$ be an observable in the algebra $\mathfrak{A}$. We consider the first $k+1$ terms of $\tau_{0}(s) \tau_{r}^{I}(\mathrm{~s}) \tau_{0}(t) S_{n+1}$, where $S_{n+1}$ denotes the first $n+1$ terms of $\tau_{r}^{I}(t) A$, and show that, for sufficiently large $n$ and $k$, this is arbitrarily close, in norm, to the sum of the first $n+1$ terms of $\tau_{0}(s+t) \tau_{r}^{I}(s+t) A$. The result follows in the limit $k, n \rightarrow \infty$.

By definition,

$$
\begin{aligned}
S_{n+1}=A+i \int_{0}^{t} d t_{1}[ & \left.V_{r}\left(t_{1}\right), A\right] \\
& +i^{2} \int_{0}^{t} d t_{1} \int_{0}^{t_{1}} d t_{2}\left[V_{r}\left(t_{1}\right),\left[V_{r}\left(t_{2}\right), A\right]\right]+\cdots(n+1 \text { terms }) .
\end{aligned}
$$

The first $k+1$ terms of $\tau_{0}(s) \tau_{r}^{I}(s) \tau_{0}(t) S_{n+1}$ are just

$$
\begin{aligned}
& \tau_{0}(s)\left\{\tau_{0}(t) S_{n+1}+i \int_{0}^{s} d s_{1}\left[V_{r}\left(s_{1}\right), \tau_{0}(t) S_{n+1}\right]\right. \\
& \left.\quad+i^{2} \int_{0}^{s} d s_{1} \int_{0}^{s_{1}} d s_{2}\left[V_{r}\left(s_{1}\right),\left[V_{r}\left(s_{2}\right), \tau_{0}(t) S_{n+1}\right]\right]+\cdots(k+1 \text { terms })\right\} \\
& =\tau_{0}(s) \tau_{0}(t)\left\{S_{n+1}+i \int_{0}^{s} d s_{1}\left[\tau_{0}^{-1}(t) V_{r}\left(s_{1}\right), S_{n+1}\right]\right. \\
& \left.\quad+i^{2} \int_{0}^{s} d s_{1} \int_{0}^{s_{1}} d s_{2}\left[\tau_{0}^{-1}(t) V_{r}\left(s_{1}\right),\left[\tau_{0}^{-1}(t) V_{r}\left(s_{2}\right), S_{n+1}\right]\right]+\cdots(k+1 \text { terms })\right\} .
\end{aligned}
$$

But $\tau_{0}^{-1}(t) V_{r}\left(s_{1}\right)=V_{r}\left(s_{1}+t\right)$, etc., and so we obtain

$$
\begin{aligned}
& \tau_{0}(s+t)\left\{A+i \int_{0}^{t} d t_{1}\left[V_{r}\left(t_{1}\right), A\right]+i^{2} \int_{0}^{t} d t_{1} \int_{0}^{t_{1}} d t_{2}\left[V_{r}\left(t_{1}\right),\left[V_{r}\left(t_{2}\right), A\right]\right]\right. \\
& +\cdots(n+1 \text { terms }) \\
& +i \int_{0}^{s} d s_{1}\left[V_{r}\left(s_{1}+t\right),\left\{A+i \int_{0}^{t} d t_{1}\left[V_{r}\left(t_{1}\right), A\right]+\cdots(n+1 \text { terms })\right\}\right] \\
& +i^{2} \int_{0}^{s} d s_{1} \int_{0}^{s_{1}} d s_{2}\left[V_{r}\left(s_{1}+t\right),\left[V_{r}\left(s_{2}+t\right),\left\{A+i \int_{0}^{t} d t_{1}\left[V_{r}\left(t_{1}\right), A\right]+\cdots\right\}\right]\right] \\
& +\cdots \\
& \left.\quad \cdots \text { up to } i^{k} \int_{0}^{s} d s_{1} \cdots \int_{0}^{s_{k-1}} d s_{k}\left[V_{r}\left(s_{1}+t\right),\left[\cdots\left[V_{r}\left(s_{k}+t\right),\{A+\cdots\}\right] \cdots\right]\right]\right\} .
\end{aligned}
$$


The total number of terms (not expanding the commutators) is $(n+1)(k+1)$. Suppose that both $n$ and $k$ are greater than, say, $m$. Then the term in " $V^{m}$ " inside the large curly bracket is

$$
\begin{aligned}
& \int_{0}^{t} d t_{1} \ldots \int_{0}^{t_{m-1}} d t_{m}\left[V_{r}\left(t_{1}\right),\left[V_{r}\left(t_{2}\right),\left[\ldots\left[V_{r}\left(t_{m}\right), A\right] \ldots\right]\right.\right. \\
& +\int_{0}^{s} d s_{1} \int_{0}^{t} d t_{1} \ldots \int_{0}^{t_{m-2}} d t_{m-1}\left[V_{r}\left(s_{1}+t\right),\left[V_{r}\left(t_{1}\right),\left[\ldots\left[V_{r}\left(t_{m-1}\right), A\right] \ldots\right]+\ldots\right.\right. \\
& +\int_{0}^{s} d s_{1} \ldots \int_{0}^{s_{j}-1} d s_{j} \int_{0}^{t} d t_{1} \ldots \int_{0}^{t_{m-j}-1} d t_{m-j}\left[V_{r}\left(s_{1}+t\right),\left[\ldots \left[V_{r}\left(s_{j}+t\right),\left[V_{r}\left(t_{1}\right),\right.\right.\right.\right. \\
& \left.\ldots\left[V_{r}\left(t_{m-j}\right), A\right] \ldots\right]+\ldots+\int_{0}^{s} d s_{1} \ldots \int_{0}^{s_{m-1}} d s_{m}\left[V_{r}\left(s_{1}+t\right),\right. \\
& {\left[\ldots\left[V_{r}\left(s_{m}+t\right), A\right] \ldots\right] .}
\end{aligned}
$$

There are $m+1$ terms (not expanding the commutators).

If we now change the variables of integration so that, for example, $\left(s_{i}+t\right)$ becomes the new $s_{i}$, and then relabel each integral so that the variables become $u_{1}, \ldots, u_{m}$ rather than $s_{1}, \ldots, s_{j}, t_{1}, \ldots t_{m-j}$, we arrive at

$$
\begin{aligned}
& \int_{0}^{t} d u_{1} \int_{0}^{u_{1}} d u_{2} \ldots \int_{0}^{u_{m-1}} d u_{m}\left[V_{r}\left(u_{1}\right),\left[V_{r}\left(u_{2}\right),[\ldots, A] \ldots\right]+\cdots\right. \\
& +\int_{t}^{s+t} d u_{1} \int_{t}^{u_{1}} d u_{2} \ldots \int_{t}^{u_{j}-1} d u_{j} \int_{0}^{t} d u_{j+1} \ldots \int_{0}^{u_{m-1}} d u_{m}\left[V_{r}\left(u_{1}\right),\left[V_{r}\left(u_{2}\right),[\ldots, A] \ldots\right]\right. \\
& +\cdots+\int_{t}^{s+t} d u_{1} \int_{t}^{u_{1}} d u_{2} \ldots \int_{t}^{u_{m-1}} d u_{m}\left[V_{r}\left(u_{1}\right),\left[V_{r}\left(u_{2}\right),[\ldots, A] \ldots\right] .\right.
\end{aligned}
$$

Adding term by term, from right to left, leads us to

$$
\int_{0}^{s+t} d u_{1} \int_{0}^{u_{1}} d u_{2} \ldots \int_{0}^{u_{m}-1} d u_{m}\left[V_{r}\left(u_{1}\right),[\ldots, A] \ldots\right]
$$

which corresponds to the $(m+1)^{\text {th }}$ term in $\tau_{r}^{I}(s+t) A$. So we see that the sum of the first $k+1$ terms of $\tau_{0}(s) \tau_{r}^{I}(s) \tau_{0}(t) S_{n+1}$ is equal to the sum of the first $n+1$ terms of $\tau_{0}(s+t) \tau_{r}^{I}(s+t) A$, (provided $k \geqq n$ ) plus a remainder consisting of a sum of certain terms in $V_{r}$ of "degree" $n+1, \ldots, n+k$. (There are, in fact, $\frac{n}{2}(n+1)+(k-n)(n+1)$ such terms.) Denote this 
remainder by $R_{n}$. Then by direct estimation of this remainder, we obtain

$$
\begin{aligned}
\left\|R_{n}\right\| \leqq & \|A\|\left\{\frac{x y^{n}}{n !}+\frac{x^{2}}{2 !}\left(\frac{y^{n-1}}{(n-1) !}+\frac{y^{n}}{3 !}\right)+\frac{x^{3}}{3 !}\left(\frac{y^{n-2}}{(n-2) !}+\frac{y^{n-1}}{(n-1) !}+\frac{y^{n}}{n !}\right)\right. \\
& +\cdots+\frac{x^{n}}{n !}\left(y+\frac{y^{2}}{2 !}+\cdots+\frac{y^{n}}{n !}\right) \\
& \left.+\left(\frac{x^{n+1}}{(n+1) !}+\frac{x^{n+2}}{(n+2) !}+\cdots+\frac{x^{k}}{k !}\right)\left(1+y+\frac{y^{2}}{2 !}+\cdots+\frac{y^{n}}{n !}\right)\right\}
\end{aligned}
$$

where we have replaced $\frac{4}{3} \pi r^{3}|s|\|V\|$ by $x$, and $\frac{4}{3} \pi r^{3}|t|\|V\|$ by $y$. The last term, viz.

$$
\left(\frac{x^{n+1}}{(n+1) !}+\cdots+\frac{x^{k}}{k !}\right)\left(1+y+\frac{y^{2}}{2 !}+\cdots+\frac{y^{n}}{n !}\right)
$$

can be made arbitrarily small (say $<\varepsilon$ ) for sufficiently large $n$ and $k$. The other terms are

$$
x \frac{y^{n}}{n !}+\frac{x^{2}}{2 !}\left(\frac{y^{n-1}}{(n-1) !}+\frac{y^{n}}{n !}\right)+\cdots+\frac{x^{n}}{n !}\left(y+\frac{y^{2}}{2 !}+\cdots+\frac{y^{n}}{n !}\right)=\Sigma,
$$

say.

Let $\varepsilon>0$ be given. Choose $m$ such that

$$
e^{y}\left(\frac{x^{m+1}}{(m+1) !}+\frac{x^{m+2}}{(m+2) !}+\cdots\right)<\varepsilon
$$

and choose $n$ so large that $n>m$ and

$$
x \frac{y^{n}}{n !}+\frac{x^{2}}{2 !}\left(\frac{y^{n-1}}{(n-1) !}+\frac{y^{n}}{n !}\right)+\frac{x^{m}}{m !}\left(\frac{y^{n-m+1}}{(n-m+1) !}+\cdots+\frac{y^{n}}{n !}\right)<\varepsilon .
$$

Then the first $m$ terms of $\Sigma$ are less than $\varepsilon$, and the remaining $n-m$ terms are less than or equal to

$$
\left(\frac{x^{m+1}}{(m+1) !}+\cdots+\frac{x^{n}}{n !}\right) e^{y}<\varepsilon, \text { i.e. } \quad \Sigma<2 \varepsilon
$$

So we have proved that $\left\|R_{n}\right\|<3 \varepsilon$, for arbitrary $\varepsilon>0$, provided $k, n$ are sufficiently large. Letting $k \rightarrow \infty$, and $n \rightarrow \infty$ gives the result.

Corollary. The homomorphisms $\tau_{r}^{I}(t)$ are automorphisms of $\mathfrak{A}$.

Proof. We need only show that the $\tau_{r}^{I}(t)$ are onto $\mathfrak{A}$. Let $B \in \mathfrak{A}$. Put $A=\tau_{0}(-t) \tau_{r}^{I}(-t) \tau_{0}(t) B$. Then $A \in \mathfrak{U}$, and $\tau_{0}(t) \tau_{r}^{I}(t) A=\tau_{0}(t) B$, which 
implies that $\tau_{r}^{l}(t) A=B$, since $\tau_{0}(t)$ is an automorphism. The $\tau_{r}^{I}(t)$ are therefore onto.

We can use the group property of $\tau_{0}(t) \tau_{r}^{I}(t)$ to give an alternative proof that $\tau_{r}^{I}(t)$ is one-one, as follows: suppose $\tau_{r}^{I}(t) A=\tau_{r}^{I}(t) B$. Applying $\tau_{0}(-t) \tau_{r}^{I}(-t) \tau_{0}(t)$ to both sides of this equation then gives the result $A=B$.

We shall now investigate $\tau_{r}^{I}(t)$ in the limit as $r \rightarrow \infty$. Let $A \in \mathfrak{U}(\mathcal{O})$ be a local observable.

Definition 1. Let $r_{0}$ be the smallest real number such that $\left[V_{\boldsymbol{a}}(t), V_{\boldsymbol{b}}(s)\right]=0$ for $|\boldsymbol{a}-\boldsymbol{b}|>r_{0}$, where $|t|<T$, and $|s|<T$.

Definition 2. Let $r_{A}$ be the smallest real number such that $\left[V_{\boldsymbol{a}}(t), A\right]=0$ for $|\boldsymbol{a}|>r_{\boldsymbol{A}}$, where $|t|<T$.

Both $r_{0}$ and $r_{A}$ are well-defined positive numbers. This follows from the fact that $A$ and $V$ are local, and Postulate 2.

Theorem 2. There exists $\delta>0$, such that for all $|t| \leqq \delta$, the limit $\tau_{r}^{I}(t) A$ exists as $r \rightarrow \infty$, for all local $A$. If we denote the limit by $A_{\infty}^{I}(t)$, then the map $A \rightarrow A_{\infty}^{I}(t)$ is continuous in $A$, and can be extended to the whole of $\mathfrak{A}$, for all $t$, and defines an automorphism of $\mathfrak{A}$ which is strongly continuous in $t$.

Proof. Let $A$ be a local observable, and let $|t|<T$. Then, according to Definitions 1 and 2, if $\left|t_{j}\right|<|t|$, and $\left|t_{k}\right|<|t|$, then

and

$$
\left[V_{\boldsymbol{a}_{j}}\left(t_{j}\right), V_{\boldsymbol{a}_{k}}\left(t_{k}\right)\right]=0 \quad \text { if } \quad\left|\boldsymbol{a}_{j}-\boldsymbol{a}_{k}\right|>r_{0}
$$

$$
\left[V_{\boldsymbol{a}_{j}}\left(t_{j}\right), A\right]=0 \text { if }\left|\boldsymbol{a}_{j}\right|>r_{\boldsymbol{A}} .
$$

Consider the general term in the series for $\tau_{r}^{I}(t) A$, namely

$$
\begin{aligned}
i^{n} \int_{0}^{t} d t_{n} \int_{0}^{t_{n}} d t_{n-1} & \ldots \int_{0}^{t_{2}} d t_{1} \int_{\left|\boldsymbol{a}_{n}\right| \leqq r} d^{3} \boldsymbol{a}_{n} \ldots \\
\cdots & \int_{\left|\boldsymbol{a}_{1}\right| \leqq r} d^{3} \boldsymbol{a}_{1}\left[V_{\boldsymbol{a}_{n}}\left(t_{n}\right),\left[\ldots,\left[V_{\boldsymbol{a}_{1}}\left(t_{1}\right), A\right] \ldots\right] .\right.
\end{aligned}
$$

Working from the inside bracket, we see that $\left[V_{\boldsymbol{a}_{1}}\left(t_{1}\right), A\right]$ is zero if $\left|a_{1}\right|>r_{A}$; similarly for the double commutator,

$$
\left[V_{\boldsymbol{a}_{2}}\left(t_{2}\right), V_{\boldsymbol{a}_{1}}\left(t_{1}\right) A\right]=\left[V_{\boldsymbol{a}_{2}}\left(t_{2}\right), V_{\boldsymbol{a}_{1}}\left(t_{1}\right)\right] A+V_{\boldsymbol{a}_{1}}\left(t_{1}\right)\left[V_{\boldsymbol{a}_{2}}\left(t_{2}\right), A\right]
$$

vanishes unless $\left|\boldsymbol{a}_{2}-\boldsymbol{a}_{1}\right| \leqq r_{0}$ or $\left|\boldsymbol{a}_{2}\right| \leqq r_{A}$. The same goes for the other term - $\left[V_{\boldsymbol{a}_{2}}\left(t_{2}\right), A V_{\boldsymbol{a}_{1}}\left(t_{1}\right)\right]$. Thus, in general, the $j$-fold commutator is zero unless $\boldsymbol{a}_{1}, \ldots, \boldsymbol{a}_{j}$ lies in a set $\left|\boldsymbol{a}_{j}\right| \leqq r_{A}$, or $\left|\boldsymbol{a}_{j}-\boldsymbol{a}_{1}\right| \leqq r_{0}$, or $\ldots$ or $\left|\boldsymbol{a}_{j}-\boldsymbol{a}_{j-1}\right| \leqq r_{0}$. We note here that $r_{0}$ is independent of $A$. 
Now, the norm of $(*)$ is bounded by

$$
\begin{aligned}
& \mid \int_{0}^{t} d t_{n} \ldots \int_{0}^{t_{2}} d t_{1} \int_{\left|\boldsymbol{a}_{n}\right|} d^{3} a_{n} \ldots \int_{\left|\boldsymbol{a}_{1}\right| \leqq r} d^{3} a_{1} \|\left[V_{\boldsymbol{a}_{n}}\left(t_{n}\right),\left[\ldots,\left[V_{\boldsymbol{a}_{1}}\left(t_{1}\right), A\right] \ldots\right] \| \mid\right. \\
& \leqq\left|\int_{0}^{t} d t_{n} \ldots \int_{0}^{t_{2}} d t_{1} \int_{S(n)} d^{3} a_{n} \ldots \int d^{3} a_{1}\left\|\left[V_{\boldsymbol{a}_{n}}\left(t_{n}\right),[\ldots, A] \ldots\right]\right\|\right|
\end{aligned}
$$

where the integrand vanishes outside $S(n)$ and its positivity means that we certainly have not decreased the value of the integral. $S(n)$ is the $3 n$-dimensional region $\bigcap_{j \leqq n} S_{j}$, where

$$
\begin{aligned}
& S_{1}=\left\{a \in \mathbb{R}^{3 n} ;\left|\boldsymbol{a}_{1}\right| \leqq r_{A}\right\}, \\
& S_{2}=\left\{a ;\left|\boldsymbol{a}_{2}\right| \leqq r_{A}\right\} \cup\left\{a ;\left|\boldsymbol{a}_{2}-\boldsymbol{a}_{1}\right| \leqq r_{0}\right\}, \\
& \cdots \cdots \ldots \ldots \ldots \ldots \ldots \ldots \\
& S_{j}=\left\{a ;\left|\boldsymbol{a}_{j}\right| \leqq r_{A}\right\} \cup\left\{a ;\left|\boldsymbol{a}_{j}-\boldsymbol{a}_{j-1}\right| \leqq r_{0}\right\} \cup \cdots \cup\left\{a ;\left|\boldsymbol{a}_{j}-\boldsymbol{a}_{1}\right| \leqq r_{0}\right\} .
\end{aligned}
$$

Expanding the commutator, we obtain as a bound for $(*)$

$$
\frac{|t|^{n}}{n !} 2^{n}\|V\|^{n}\|A\| \int_{S(n)} d^{3} a_{n} \ldots \int d^{3} a_{1}
$$

The integral over $S(n)$ can be split up into $n$ ! parts (not all disjoint), each of the form of a polysphere in suitable coordinates, namely

$$
\left|\boldsymbol{a}_{1}\right| \leqq r_{A}, \quad\left|\boldsymbol{a}_{2}^{\prime}\right| \leqq \text { const., } \quad\left|\boldsymbol{a}_{3}^{\prime}\right| \leqq \text { const., } \ldots
$$

where $\boldsymbol{a}_{j}^{\prime}$ is either $\boldsymbol{a}_{j}$, when the constant is $r_{A}$, or is one of $\boldsymbol{a}_{j}-\boldsymbol{a}_{k-1}$, $(2 \leqq k \leqq j)$, when the constant is $r_{0}$.

For each $j \in(1, \ldots, n)$ let us put

say, and

$$
\alpha_{j, 1}=\int_{\left|a_{j}\right| \leqq r_{A}} d^{3} a_{j}=\frac{4}{3} \pi r_{A}^{3}=D
$$

$$
\alpha_{j, k}=\int_{\left|a_{j}-\boldsymbol{a}_{k-1}\right| \leqq r_{0}} d^{3}\left(a_{j}-a_{k-1}\right)=\frac{4}{3} \pi r_{0}^{3}=d
$$

say, for $k=2, \ldots, j$.

Then the typical term in the integral over $S(n)$ is

$$
\alpha_{1,1} \alpha_{2, i_{2}} \ldots \alpha_{n, i_{n}} \text { where } i_{j} \in\{1, \ldots, j\} \text {. }
$$

The integral over $S(n)$ is therefore bounded above by the sum

$$
\sum \alpha_{11} \alpha_{2 i_{2}} \ldots \alpha_{n, i_{n}}
$$


(where the sum is over all possible values of $i_{2}, i_{3}, \ldots, i_{n}$ )

$$
=D(D+d)(D+2 d) \ldots(D+(n-1) d) .
$$

It follows, then, that $(*)$ is bounded in norm by

$$
\frac{|t|^{n}}{n !} 2^{n}\|V\|^{n}\|A\| D(D+d) \ldots(D+(n+1) d) \text {. }
$$

Let $r$ and $r^{\prime}$ be so large that the first $N$ terms of $\tau_{r}^{I}(t) A$ and $\tau_{r^{\prime}}^{I}(t) A$ are the same. This is possible since each $S(n)$ is a bounded region, and consequently, as $r$ increases, each term of $\tau_{r}^{I}(t) A$ becomes independent of $r$. Then

$$
\left\|\tau_{r}^{I}(t) A-\tau_{r^{\prime}}^{I}(t) A\right\| \leqq 2 \sum_{n=N}^{\infty} \frac{|t|^{n}}{n !} 2^{n}\|V\|^{n}\|A\| D \ldots(D+(n-1) d) .
$$

The series $\sum_{n} \frac{|t|^{n}}{n !} 2^{n}\|V\|^{n} D(D+d) \ldots(D+(n-1) d)$ converges, uniformly in $|t| \leqq \delta$, by the ratio test, for $\delta<1 / 2\|V\| d$. Hence, given $\varepsilon>0$, for sufficiently large $N$, (i.e. sufficiently large $r, r^{\prime}$ )

$$
\left\|\tau_{r}^{I}(t) A-\tau_{r^{\prime}}^{I}(t) A\right\|<\varepsilon .
$$

The $\tau_{r}^{I}(t) A$ therefore form a Cauchy net, and hence converge in $\mathfrak{A}$.

The $\delta$ defined above depends only on $V$ and $r_{0}$, not on $A$. It follows that, for $|t| \leqq \delta, \tau_{r}^{I}(t) A$ converges uniformly as $r \rightarrow \infty$, for all local $A$. Since each $\tau_{r}^{I}(t) A$ is continuous in $A$, it follows that $\tau_{\infty}^{I}(t) A=A_{\infty}^{I}(t)$ is also continuous in $A$, for $A$ local, and $|t| \leqq \delta$. We can therefore extend $\tau_{\infty}^{I}(t)$, for $|t| \leqq \delta$, by continuity, to the whole of $\mathfrak{A}$.

Now, $\tau_{\infty}^{I}(t)$ is the strong limit of automorphisms, and is therefore certainly a ${ }^{*}$-homomorphism. We shall show that $\tau_{\infty}^{I}(t)$ is one-one and onto, for $|t| \leqq \delta$. The relation

$$
\tau_{0}(s) \tau_{r}^{I}(s) \tau_{0}(t) \tau_{r}^{I}(t)=\tau_{0}(s+t) \tau_{r}^{I}(s+t), \quad \text { for } \quad|s|<\delta,
$$

$|t|<\delta$, implies that $\tau(s) \tau(t)=\tau(s+t)$, where $\tau(t)$ denotes $\tau_{0}(t) \tau_{\infty}^{I}(t)$. We deduce that $\tau_{\infty}^{I}(t)$ is an automorphism, as in the corollary to Theorem 1 .

So far we have defined a family of automorphisms, $\tau(t)$, with $|t| \leqq \delta$. We can extend these to all $t$.

Define, for $|\theta| \leqq 2 \delta$,

$$
\tau(\theta)=\tau(x) \tau(y),
$$


where $|x| \leqq \delta,|y| \leqq \delta$, and $x+y=\theta$. The right hand side is well-defined, and is an automorphism of $\mathfrak{A}$. If $|\theta| \leqq \delta$, this definition is no more than an identity. Suppose $\delta<|\theta| \leqq 2 \delta, x+y=\theta=x^{\prime}+y^{\prime},|x|,\left|x^{\prime}\right|,|y|,\left|y^{\prime}\right|<\delta$, and $x \neq x^{\prime}$. Then

$$
\begin{aligned}
\tau(x) \tau(y) & =\tau\left(x^{\prime}\right) \tau\left(x-x^{\prime}\right) \tau(y) \\
& =\tau\left(x^{\prime}\right) \tau\left(y^{\prime}-y\right) \tau(y), \quad \text { using } \quad x-x^{\prime}=y^{\prime}-y, \\
& =\tau\left(x^{\prime}\right) \tau\left(y^{\prime}\right),
\end{aligned}
$$

which simply means that $\tau(\theta)$ is independent of how we write $\theta=x+y$, i.e. is well-defined. We have therefore extended the range of $t$, from $|t| \leqq \delta$, to $|t| \leqq 2 \delta$. In this way we can define $\tau(t)$ for any $t$.

That $\tau_{\infty}^{I}(t)$ is strongly continuous in $t$ follows from the obvious fact that $\tau_{r}^{I}(t)$ is strongly continuous for each $r$, and the following estimate:

$$
\begin{aligned}
\left\|\tau_{\infty}^{I}(t) A-\tau_{\infty}^{I}\left(t^{\prime}\right) A\right\| & \leqq\left\|\tau_{\infty}^{I}(t) A-\tau_{r}^{I}(t) A\right\| \\
& +\left\|\tau_{r}^{I}(t) A-\tau_{r}^{I}\left(t^{\prime}\right) A\right\|+\left\|\tau_{r}^{I}\left(t^{\prime}\right) A-\tau_{\infty}^{I}\left(t^{\prime}\right) A\right\| .
\end{aligned}
$$

The first and third terms on the r.h.s. can be made arbitrarily small, for sufficiently large $r$ (convergence is uniform in $t$ ), and the second for $\left|t-t^{\prime}\right|$ sufficiently small. This proves the theorem.

Remark. The family $\tau_{0}(s) \tau_{\infty}^{I}(s) \equiv \tau(s)$ is a one-parameter group of automorphisms of $\mathfrak{A}$, strongly continuous in $s$, for all $s$.

Theorem 3. $\tau_{\infty}^{I}(t)$ commutes with space translations, i.e. $\tau_{\infty}^{I}(t) \tau_{0}(0, \boldsymbol{a})$ $=\tau_{0}(0, \boldsymbol{a}) \tau_{\infty}^{I}(t)$, for all $\boldsymbol{a}$.

Proof. Let $A$ be local, and $|t|<\delta$; then we have

$$
\begin{aligned}
\tau_{0}(\boldsymbol{a}) \tau_{\infty}^{I}(t) A & =\tau_{0}(\boldsymbol{a})\left(A+i \int_{0}^{t} d t_{1} \int d^{3} a_{1}\left[V_{\boldsymbol{a}_{1}}\left(t_{1}\right), A\right]+\cdots\right) \\
& =\tau_{0}(\boldsymbol{a}) A+i \int_{0}^{t} d t_{1} \int d^{3} a_{1}\left[V_{\boldsymbol{a}_{1}+\boldsymbol{a}}\left(t_{1}\right), \tau_{0}(\boldsymbol{a}) A\right]+\cdots \\
& =\tau_{\infty}^{I}(t) \tau_{0}(\boldsymbol{a}) A
\end{aligned}
$$

Continuity of the automorphisms gives the result for all $A \in \mathfrak{A}$. It remains to remove the restriction $|t| \leqq \delta$. This is easily done as follows; let $s$ be given. Then there exists $t$, with $|t| \leqq \delta$, and a positive integer $m$ such that $m t=s$. Let $A \in \mathfrak{A}$. 


$$
\begin{aligned}
\tau_{0}(0, \boldsymbol{a}) \tau_{\infty}^{I}(s) A & =\tau_{0}(-s) \tau_{0}(0, \boldsymbol{a}) \tau_{0}(s) \tau_{\infty}^{I}(s) A \\
& =\tau_{0}(-s) \tau_{0}(0, \boldsymbol{a})(\tau(t))^{m} A=\tau_{0}(-s)(\tau(t))^{m} \tau_{0}(0, \boldsymbol{a}) A \\
& =\tau_{\infty}^{I}(s) \tau_{0}(0, \boldsymbol{a}) A, \quad\left(\text { where } \tau(t)=\tau_{0}(t) \tau_{\infty}^{I}(t)\right)
\end{aligned}
$$

This completes the proof of the theorem.

Clearly, both Theorem 2 and Theorem 3 hold in any number of dimensions.

If, in addition to the assumptions 1,2,3, we have a continuous representation of $0(3)$ in Aut $\mathscr{U}$, and if we choose $V$ to be invariant under this action of $0(3)$, the theory will be Euclidean invariant.

Suppose that instead of a general $B^{*}$-algebra $\mathfrak{A}$, we consider the algebra constructed as follows. Define $\mathscr{R}(\mathcal{O})$ to be the $W^{*}$-algebra generated by $\phi(f), \pi(g)$ as $f, g$ run over $\mathscr{D}(\mathcal{O})$, where $(\phi, \pi)$ is the usual relativistic scalar free field at time $t=0$ and where now $\mathscr{O} \subset \mathbb{R}^{3}$; let $\mathfrak{A}_{0}$ be the $C^{*}$-algebra generated by the $\mathscr{R}(\mathcal{O})$. Then results worded as our Theorems 2 and 3 , with $\mathfrak{U}_{0}$ replacing $\mathfrak{A}$, can be proved by similar methods (using weak integrals instead of Riemann-Bochner integrals). (But in this case, $\tau_{0}(t)$ will not be continuous in $t$ in the sense of assumption 3 , so that the continuity of the time evolution, $\tau_{0}(t) \tau_{\infty}^{I}(t)$, will be a representation-dependent concept.)

If $A \in \mathscr{R}(\mathcal{O})$ and $f \in \mathscr{D}(\mathcal{O})$, it is easy to prove from our estimates that the norm limit, $\tau(t) A$, of $\tau_{r}(t) A=\tau_{0}(t) \tau_{r}^{I}(t) A$ as $r \rightarrow \infty$, is uniform in $\|A\| \leqq 1$. Since $\tau_{r}(t)$ is implemented, it follows that $\tau_{r}(t) \mathrm{e}^{i \alpha \phi(f)}$ and $\tau_{r}(t) e^{i \alpha \pi(f)}$ are strongly continuous in $\alpha$, and since the limits $r \rightarrow \infty$ are uniform in $\alpha$, we see that $\tau(t) e^{i \alpha \phi(f)}$ and $\tau(t) \mathrm{e}^{i \alpha \pi(f)}$ are strongly continuous in $\alpha$. This means that the sharptime Heisenberg fields $\phi(f, t)$, $\pi(f, t)$ can be defined as the self-adjoint generators

$$
\phi(f, t)=\left.\frac{d}{d \alpha} \tau(t) e^{i \alpha \phi(f)}\right|_{\alpha=0},
$$

the limit being strong on the domain $D_{t}$ of $\phi(f, t)$. In general, $D_{t}$ will depend on $t$.

The resulting theory fails to be satisfactory because

(1) time-evolution is not implemented,

(2) locality and relativity do not hold.

The first would be remedied by changing to a new representation, as required by Haag's theorem [7]. The second is due to the non-local nature of the interaction.

Note added in proof. Bounded interactions without a space cut-off have been considered by Høegh-Krohn as weak limits of cut-off theories (see Boson Fields under a General Class of Local Relativistic Invariant Interactions); and by Efimov, and others in perturbation theory. (See A. Salam, R. Delbourgo and J. Strathdee, Phys. Rev. 187, 1999 (1969) and references therein.) 


\title{
References
}

1. Segal, I. E.: Quasi-finiteness of the interaction Hamiltonian of certain quantum fields. Ann. Math. 72, 594 (1960).

2. Haag, R.: Colloques sur les problèmes mathématiques de la théorie quantique des champs. Paris: Centre Nationale des Recherches Scientifiques 1959; - Haag, R., Schroer, B.: Postulates of quantum field theory. J. Math. Phys. 3, 248 (1962); Haag, R., Kastler, D.: An algebraic approach to quantum field theory. J. Math. Phys. 5, 848 (1964).

3. Guenin, M.: On the interaction picture. Commun. Math. Phys. 3, 120 (1966).

4. Streater, R. F.: The Heisenberg ferromagnet as a quantum field theory. Commun. Math. Phys. 6, 233 (1967).

5. - On certain non-relativistic quantized fields. Commun. Math. Phys. 7, 93 (1968).

6. See Yosida, K.: Functional analysis, p. 132. Berlin-Heidelberg-New York: Springer 1965.

7. Haag, R.: On quantum field theory. Dan. Mat. Fys. Medd. 29, 12 (1955).

8. Robinson, D. W.: Commun. Math. Phys. 7, 337 (1968).

9. Ruelle, D.: Statistical mechanics. New York: Benjamin 1969.

\author{
R. F. Streater \\ I. F. Wilde \\ Mathematics Department \\ Bedford College \\ Regent's Park \\ London, N.W. 1, Great Britain
}

